
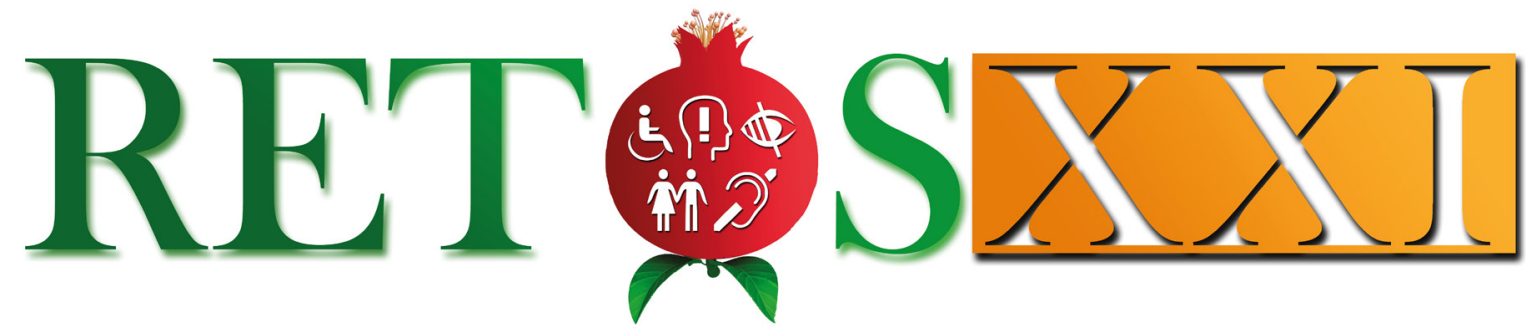

LA NECESIDAD DE INVESTIGACIÓN-ACCIÓN COMO MEJORA EDUCATIVA THE NEED FOR RESEARCH-ACTION AS AN EDUCATIONAL IMPROVEMENT

\title{
SORDEDAD Y SORDERA: SU IMPLICACIÓN EN LA CULTURA Y EN EL DESARROLLO EVOLUTIVO PSICO Y SOCIOLINGÜÍSTICO EN LA EDUCACIÓN BILINGÜE
}

\section{SORDINNESS AND DEAFNESS: ITS INVOLVEMENT IN CULTURE AND EVOLUTIONARY DEVELOPMENT PSYCHO AND SOCIOLINGUISTIC IN BILINGUAL EDUCATION}

NOEL DANIEL

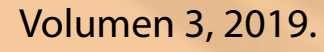

Aceptado: 6/07/2019 
SORDEDAD Y SORDERA: SU IMPLICACIÓN EN LA CULTURA Y EN EL DESARROLLO EVOLUTIVO PSICO Y SOCIOLINGÜÍSTICO EN LA EDUCACIÓN BILINGÜE

SORDINNESS AND DEAFNESS: ITS INVOLVEMENT IN CULTURE AND EVOLUTIONARY DEVELOPMENT PSYCHO AND SOCIOLINGUISTIC IN BILINGUAL EDUCATION

NOEL DANIEL 1

1 Especialista en educación especial, universidad especializada de las américas, ndanisaquiles@gmail.com. 


\section{Resumen}

La "sordera", como visión de un modelo clínico que se tiene de los sordos, en contraste con la "sordedad", la cual es la realidad como se miran estas personas así mismas, genera un profundo análisis de ambas perspectivas y su implicación en la cultura y en el desarrollo evolutivo, psico y sociolingüístico que asegure una educación bilingüe a esta población. Los aportes señalados por Martínez (2008) permiten comprender la importancia de la adquisición de la lengua (Lengua de señas, lengua escrita, lengua oral); la lengua es una de las manifestaciones socio culturales y psicoevolutiva evidentes de la superioridad del hombre. Estos argumentos hacen un llamado a los lectores, para que profundicen en los estudios lingüísticos, psicolingüísticos, sociolingüísticos y antropológicos que surgen de ambas perspectivas: lo clínico y lo social. Adicionalmente, este documento muestra la comprensión de la naturaleza humana, sus avances y evolución sin importar que una condición física (sordera) prive al individuo (sordedad) de su aprendizaje.

Palabras clave: sordera, sordedad, sordo, cultura de sordos, desarrollo evolutivo y sociolingüístico, educación de sordos, educación bilingüe.
"Deafness", as a vision of a clinical model of the deaf, in contrast to the "sorfness", which is the reality as these people look themselves, generates a deep analysis of both perspectives and their involvement in culture and development evolutionary, psychoanalytic and sociolinguistic that ensures a bilingual education this population. The contributions pointed out by Martínez (2008) allows understand the importance of acquisition of the language (Sign Language, Written Language, Oral Language); the language is one of the evident socio-cultural and psycho-evolutionary manifestations of man's superiority. These arguments call upon readers to delve deeper into linguistic, psycholinguistic, sociolinguistic and anthropological studies that arise from both perspectives: the clinical and the social. In addition, this document shows the understanding of human nature, its advances and evolution regardless of whether a physical condition (deafness) deprives the individual (sordid) of his learning.

Keyword: Deafness, deafness, deaf ness, evolutionary and sociolinguistic development, education of the deaf, bilingual education. 


\section{INTRODUCCIÓN}

La "British Broadcasting Corporation (por sus siglas en inglés, BBC)", Corporación Británica de Radiodifusión, la cual es el servicio público de radio, televisión e internet de Reino Unido, con más de nueve décadas de trayectoria, reporta en el portal de su página web, que la Organización Mundial de la Salud "estima que 360 millones de personas sufren pérdida auditiva, 328 millones de adultos y 32 millones de niños, más del $5 \%$ de la población mundial" (OMS, 2013, p. s/n). Según el organismo, tienen "pérdida auditiva aquellas que oyen menos de 40 decibelios con el oído bueno, en el caso de los adultos, y 30 decibelios en el caso de los niños" (BBC, 2014, p. s/n). De acuerdo con esta información, "la mayoría de estas personas viven en países de renta baja y media. Aproximadamente, un tercio de las personas mayores de 65 años se ven afectados por la pérdida auditiva, dice la Organización Mundial de la Salud. La prevalencia en este grupo de edad es mayor en el sur de Asia, en el Pacífico asiático y en el África subsahariana" (BBC, 2014, p. s/n). Esta información presentada por la Corporación "BBC" responde a un enfoque clínico que hace referencia a la sordera.

Sin embargo, el sitio web de la World Federation for the Deaf "WFD"establece que existen en el mundo 70 millones de personas sordas. Un porcentaje no determinado de ellas tiene la lengua de señas como su principal medio de comunicación, y con ella, también, una cultura (WFD, 2018, p. s/n) propia evidenciada por una comunidad, bien definida, que se distingue en el contexto como un grupo minoritario dentro de las comunidades mayoritarias oyentes en las cuales los sordos habitan (Daniel, 2016). Este dato, registrado por la "WFD", responde a un enfoque de sordedad.
Adicional a ello, la cosmovisión que tienen los sordos de sí mismos considera que ellos, como grupo, forman parte de una comunidad sorda que comparte una lengua común, experiencias y valores comunes y una forma común de interactuar entre ellos y con las personas oyentes (Baker y Padden, (1978, p. 4). De lo cual, López (2018) explica, con lujo de detalles, en la clase de Cultura de Sordos, en una de sus disertaciones, señalando una interesante postura: "De la sordera a la sordedad hay una persona sorda de diferencia" (López, 2018, s/n).

\section{PERSPECTIVA LÉXICO- CONCEPTUAL}

La sordera y la sordedad son conceptos que definen perspectivas diferentes y que conjugan la visión que tienen, respectivamente, las personas oyentes acerca de las personas sordas (sordera) y las personas sordas de sí mismos (sordedad). Estos conceptos, según Howard (2017), no pueden ser vistos como un estado finito, sino como procesos de evolución, por los cuales tanto las personas oyentes como las personas sordas han concebido en oposición la identidad sorda y su dura historia. Historia definida como un cuento contado por los benefactores oyentes, pero que, Harlan Lane (1984), en su libro: "When de mindhears" (cuando la mente escucha), la define como una historia falsa contada por oyentes (Lane, 1984). Después de siglos de transmisión oral, son los propios sordos quienes reformulan la sordera a la luz de la sordedad para darle sentido a su tradición.

La sordedad conforma un postulado que dirige el pensamiento que los individuos sordos, en construcción de esa identidad, emplean alrededor de un conjunto ordenado de prioridades y principios, que son afectados por diversos factores como la nación, la época, la clase y la lengua. Evidencias 
estas que definieron, por muchos siglos, la perspectiva histórica y sociológica de las vidas de las personas sordas en el mundo (Howard, 2016). Lo que también examina las implicaciones históricas sobre las prácticas patológicas seguidas y que, en la actualidad, en algunos países continúan utilizándose en la prestación de servicios y en la educación para personas sordas, especialmente en países latinoamericanos. Por ello, el reconocimiento de la identidad de sordo conduce a la necesidad de cambiar dicha perspectiva sociológica asegurando que las comunidades de sordos en el mundo conforman una minoría lingüística y cultural, pero con una riqueza incalculable que les ubica (al sordo) fuera del contexto de persona con discapacidad.

\section{PERSPECTIVA HISTÓRICA}

En la concepción sobre sordera y la discapacidad puede observarse su evolución detenidamente durante el proceso educativo en el que ha participado el sordo a lo largo de su historia, a quien su educación puede considerársele desde dos puntos de vista. Uno tradicional, en el cual los niños sordos fueron catalogados desde el punto de vista médico como niños con discapacidad, que surge como consecuencia de la interacción entre una dificultad específica para percibir a través de la audición los sonidos del ambiente y dependiendo del grado de pérdida auditiva (Román, 2007) pues, carecen de la capacidad para oír la lengua hablada (Veinberg, 2007). No obstante, este planteamiento es una perspectiva que no engloba la realidad absoluta.

Para entender la sordedad, es necesario ahondar en el pasado y realizar una revisión interesante sobre temas de cultura, enculturación y aculturación, haciendo una explicación de las diferencias y su comparación con el pensamiento occidental (eurocentrismo) y no occidentales de la definición de 'cultura', su evolución histórica y cómo se llega a la definición de 'cultura sorda', incluyendo el idioma y la lengua de señas (lingüísticamente); así como comprender que la 'cultura' define y categoriza a las comunidades sordas como una entidad única (Howard, 2016). Este punto de vista lleva a la reflexión del cruel proceso de evolución experimentado por la comunidad sorda panameña, de sus luchas libradas durante la década de los 80 's, cuando se les exigía hablar lengua oral y prohibía hablar por medio de su lengua natural, la Lengua de Señas.

A lo largo de la propia experiencia personal como oyente, en la comunidad sorda, aprendí que una persona sorda, si se acepta tal y como es, constituye una de las principales condiciones para el desarrollo de su existencia, vida y sentido del "ser". Pues lo contrario identifica a la persona con una discapacidad auditiva, enfatiza la pérdida auditiva, lo hace diferente y condiciona su normalidad. Lo que obviamente se sabe: un sordo y un oyente son diferentes. Pero, su diferencia, más que física, radica en su formación de conceptos y no necesariamente en su capacidad para aprender. La modalidad en que se da el proceso de desarrollo de pensamiento sí puede que los haga diferentes. Existe una modalidad con orientación visual gestual para el aprendizaje del sordo, mientras que existe el aprendizaje del oyente ocurre por una modalidad vocal auditiva (Daniel, 2006). Es más, Alisedo (1998) indica que para que se dé el lenguaje tienen que existir tres dimensiones sine quanon: cerebro humano neurológicamente sano, entorno lingüístico y comunicación dialógica.

El pretender o querer que un sordo aprenda mediante la modalidad vocal auditiva podría colocarlo en cierta desventaja. La sordedad enfatiza que, en este caso, la diferencia del "mundo" sordo con la oyente muestra a ambos 
individuos (sordos - oyentes) como sujetos separados, los que hablan y los que no hablan. Pero ¿cómo un sordo que utiliza su lengua de señas puede ser catalogado que no habla?, si mediante su lengua natural, expresa sus ideas, pensamiento, sentimientos y demás componentes característicos de las lenguas. Peor aún, para el caso del sordo, colocarlo dentro o siendo 'parte' del mundo oyente, es hacerlo dependiente de la audición, la cual por naturaleza anatómica registra en su condición una limitación (Howard, 2016).

\section{PERSPECTIVA EPISTEMOLÓGICA}

Por otra parte, la epistemología de la sordera ve a la lengua de señas en el mundo como una forma de comunicación, pero ¿realmente es un verdadero idioma? Sí, pero condicionado a la lengua oral. De allí puede recordarse "la famosa Comunicación Total", "el CuedSpeech" y otras formas bimodales, que a criterio de lo que enseña la sordedad fueron experiencias del pasado. No obstante, la identidad cultural sorda afronta la naturaleza y extensión del conocimiento de la persona sorda, desde su aceptación de ser sordo. Su sordedad la adquiere al nacer, crecer y vivir en una comunidad lingüística minoritaria sin tener en cuenta que la base principal sea la audición. Ha quedado evidenciado que una persona puede explorar la vida sin la audición y cuanto más rápido sea aceptada su condición mejor serán los resultados que se obtienen en la vida y en la educación de estas personas.

No es necesario que un sordo sea tratado como un oyente, ni utilice audífonos, que, muchas veces, no aportan valor agregado. Por el contrario, afectan su autovaloración. Lo mejor es aceptar que la diferente percepción del mundo, con base a la perspectiva visual, les valida su autoconcepto y autodeterminación "como persona sorda".
La posesión de una educación, cultura, lengua e identidad (sordos vs disminución de la audición y otros) hace que la persona crezca sin preconceptos y limitación, que han sido catalogados como aspectos patológicos y sociológicos, adentrarse a la definición y diferencias de 'discapacidad' y al criterio de "minoría cultural"; así como también, a la percepción social. Howard (2016) lo define como "simulacrumisation" (imagen y representación de las mayorías), es la percepción basada en la patología (medicalización de ser sordo y la educación). Esta visión patológica explica la discusión sostenida en el congreso de Milán de 1880 y la nueva era: que involucra la participación de sordos; luego de su participación y la colaboración de 2010, cuando los sordos retoman el destino de su propio devenir histórico y futuro, se hacen responsable de su postura a nivel global (crean un impacto social). Es decir, mientras que la sordera avergüenza al sordo, la sordedad lo llena de orgullo, le otorga patrimonio y espacio en construcción de su verdadera historia e identidad, que son tan necesarias para su renacimiento.

\section{PERSPECTIVA LINGÜÍSTICA}

Con el fin de documentar lo anteriormente planteado, este escrito enfatiza la importancia de la adquisición de la lengua, siendo que la lengua es una de las manifestaciones socio culturales y psicoevolutivas evidente de la superioridad del hombre. Pero, a partir de este momento, puntualiza ambas perspectivas, de la sordera y la sordedad, para que los lectores saquen sus propias conclusiones con base a estudios lingüísticos, psicolingüísticos, sociolingüísticos y antropológicos. Este documento intenta la comprensión de la naturaleza humana, sus avances y evolución sin importar que una condición física prive al individuo de su aprendizaje.

Aunque no es la única vía para la 
comprensión del ser humano, las experiencias y vivencias planteadas por grandes teóricos y exponentes, de la talla de Chomsky, Vigostky, Piaget y Lenneberg, entre otros, quienes llevan a la reflexión, por medio de sus estudios, de que todo cuanto piensa el hombre se plasma en un código representado mediante símbolos y signos, que desde los primeros estadios de la vida, luego del nacimiento, la humanidad adquiere o desarrolla tan compleja capacidad propia para expresar pensamientos y sentimientos por medio de la palabra. Stokoe (1960) lo fundamenta en la estructuración de la lengua de señas, como una lengua natural. Quiere decir que si bien es cierto que el hombre acumula el conocimiento en su mente y lo registra de manera sorprendente para toda la vida, lo mismo ocurre en cualquier comunidad, donde para comunicarse se requiera la modalidad oral, la escritura o los gestos y las señas. Todas las lenguas, visuales, escritas, orales y de señas son patrimonio de la humanidad.

Martínez (2008) realiza una explicación de tópicos vinculados al desarrollo cognitivo, psicomotor y socio afectivo del niño, pero lo lleva a una condición de discapacidad auditiva con implicaciones en su educación, lo que indiscutiblemente se desvincula de la perspectiva social y a la sordedad, al corresponderse con un enfoque clínico. Pero como se quiere vincular a la sordedad también debe analizarse la compresión del desarrollo evolutivo y su implicación para la educación del sordo desde una visión sorda. No soy sordo, soy oyente con muchos años dentro de la comunidad señante, no soy parte de la comunidad sorda, porque no tengo familia sorda, pero por mi afinidad por mi enculturación sorda me permito emitir opinión sobre lo que a juicio de los expertos constituye la sordedad.

Para Rodríguez (2008) es evidente que, para la perspectiva oralista, la evolución que se logra en el tratamiento de la deficiencia o discapacidad auditiva, al igual que en muchas otras patologías infantiles, está condicionada en gran medida a la rapidez con la que se traten. Por ello, una buena detección sentará las bases de un determinado nivel de desarrollo del lenguaje hablado oralmente en un niño "normal" pero no así en el caso de los sordos. Stokoe demostró que la intervención e interacción temprana del niño sordo con su lengua de señas contribuye al desarrollo de su nivel cognitivo. Adicional a ello, Peluso y Vallarino (2015, citado en Sánchez, 2016, p.148) afirma que "los métodos de educación para sordos, en lo que tiene que ver con el uso o no de lenguas de señas y lenguas orales, pueden agruparse en cuatro tipos: el oralismo, que implica la organización de grupos de sordos para ser educados exclusivamente mediante la lengua oral; el bimodalismo, que supone el uso indiscriminado y desorganizado de ambas lenguas a la vez; el neo oralismo, que integra a los sordos a clases oyentes (siendo más eficiente que el oralismo clásico en su erradicación de la lengua, ya que trunca la formación de pequeñas comunidades de sordos en los salones de clase); y el bilingüismo, que se caracteriza por integrar ambas lenguas de forma discriminada y organizada, como primera y segunda lengua del alumno" (p.148).

Lo anteriormente expuesto va en contra de la perspectiva de sordedad. No es lo mismo. El desarrollo psicoevolutivo de los niños sordos depende de muchos factores, el grado de la pérdida auditiva, la etiología de la misma, el nivel intelectual del sujeto, la existencia de restos auditivos, la colaboración e implicación familiar, si el sujeto ha recibido atención $\mathrm{o}$ no temprana y tratamiento logopédico, implantación de prótesis auditivas, etc. Pero no son determinantes absolutas para el aprendizaje del sordo, pues los sordos aprenden excelentemente bien mediante la lengua de señas. Las indicaciones señaladas 
al inicio de este párrafo son señalamientos comunes de una perspectiva clínica que persigue la atención y detección temprana de la condición desde los primeros estadios de vida del niño.

Ahora bien, se reconoce en estudios realizados que la competencia cognitiva de los sordos es semejante a la de los oyentes, aunque la actuación en pruebas de evaluación cognitivas sí sea menor que la de los niños con audición normal. Esto se debe a que muchas veces las pruebas no han sido estandarizadas para esta población y que el desconocimiento de una lengua visual gestual por parte de los especialistas evaluadores parcializa los resultados hacia una respuesta de deficiencia. Estoy seguro de que si los especialistas hablan en lengua de señas los resultados serían diferentes, pues la implicación de una lengua de señas, natural, asegura que la aplicación de dichas pruebas se ajuste a la realidad del sujeto.

A mi juicio, si un niño sordo es evaluado en una lengua oral, obviamente, estará en desventaja con respecto a sus pares. Pero si se ajustan los criterios a una lengua de señas los resultados obtenidos de dichos instrumentos de evaluación serán más favorables para la persona sorda. Este planteamiento ha sido sometido a la investigación internacional, pero debe someterse a la experimentación nacional para que se evidencie que el desarrollo psicoevolutivo del niño sordo depende de un conjunto de factores, entre los cuales se encuentran: padres comprometidos con la lengua de señas, la existencia de pautas para un desarrollo lingüístico y comunicativo que permita la aculturación del sordo y le potencia para que tenga la oportunidad de aprender en la lengua natural, visual y gestual, dadas sus limitaciones auditivas.

Es obvio que la visión antropológica lingüística demuestra que si los profesionales imparten clase a los niños sordos en su lengua y cultura (si estos profesionales además son sordos), los niños sordos lograran establecer esos vínculos necesarios para que lleguen a la abstracción. Es más, si se parte de la concepción de sordedad, y el mundo fuese al revés, "que todas las personas fueran sordas" y que lo excepcional fuera ser oyente, a los oyentes no le costaría mucho adaptarse a la visión de la sordedad, puesto que poseen la audición. Quiere decir que la sordedad impulsa la formación en lo visual. Los niños sordos y oyentes ambos pueden ver.

Lo anteriormente analizado evidencia la cruda realidad de la situación del sordo en muchos países, donde aún se aprecia la condición de la sordera y no desde la sordedad. Pues la sordedad considera que la visión del mundo desde la perspectiva del sordo siempre va ser diferente. Los sordos profesionales han tenido que imbuirse en el mundo oyente y descubrir mediante investigaciones que el desarrollo psicoevolutivo, la formación de concepto y el aprendizaje del niño sordo difiere al del oyente, por razones de modalidad o de la sustancia: los oyentes aprenden y evolucionan su lengua mediante una modalidad vocal auditiva, mientras que en las personas sordas la evolución de su lengua y aprendizaje se realiza mediante la modalidad visual - gestual.

\section{PERSPECTIVA EDUCATIVA}

En otros artículos, escrito por mí, he explicado lo aprendido en otras clases como, Lingüística, Psicolingüística y Cultura de Sordos, con respecto a la educación de sordos, su evolución y procesos implicados en esta experiencia. Porque la adecuación y el abordaje de la educación de sordo, en los países en vía de desarrollo, es una cuestión complicada. Según algunos expertos, la sociedad, la academia y el sistema educativo 
tienen que hacer posible el acceso del sordo a los escenarios de actuación, para que se constituyan en formadores de sus pares y homólogos sordos bajo una perspectiva socio antropológica, que identifica la visión del sordo como ser sociolingüístico con representación social, opuesta a la visión del modelo clínico oral (Veinberg, 2002).

No puede soslayarse que han existido componentes escolarizados sordos en la metodología oral con buenos resultados, pero esta salvedad es apreciable en situaciones donde la pérdida auditiva genera una hipoacusia que permite que el alumno interactúe en el mundo oyente, con ciertas limitaciones, pero sin grandes repercusiones. No obstante, debe considerarse la opinión y el interés de la población, quienes, desde su sordedad, aspiran a una sociedad que acepte su condición y reproduzca soluciones que garantice su pleno desarrollo psicoevolutivo $y$ social.

Como lo plantee en otros escritos sobre Cultura de Sordos, y haciendo eco de las palabras de la docente que impartió la clase y que es una persona sorda, a los sordos debe permitírsele el acceso a la educación superior y la participación ciudadana y colectiva que aseguren la implantación de un modelo social con enfoque cultural antropológico y lingüístico, desde la perspectiva de la sordedad (López, 2018).

A lo largo de mi experiencia educativa y de la ruta que tracé en mi vida, he sido partícipe en luchas sostenidas por sordos nacionales e internacionales, quienes abogan por la construcción de un modelo social, que asegure, además de su participación plena en una sociedad plural, el derecho a una educación bilingüe, lo mismo que el reconocimiento de la lengua de señas.
Mientras que, en otra clase, se estudió el libro de Psicolingüística CECAR (2018), en el cual plantea al lenguaje como un instrumento del espíritu humano, un producto estático y cerrado en sí mismo con su propia historia, constituye un sistema de signos subordinado al mundo prelingüístico "dado" primariamente y que lo reproduce sin distorsión alguna (CECAR, 2018). Cabe destacar que este argumento es acorde al desarrollo "normal" psicoevolutivo del hombre. Pero en el caso de la sordera, aun cuando existe un mundo prelingüístico y que se desarrolla visualmente, si se observa desde la perspectiva oral, para el niño sordo, se convierte en una experiencia distorsionada de la realidad.

La respuesta educativa para la persona sorda ha tenido su basamento en dos corrientes educativas, la oralista (Lengua Oral) y la manualista (Lengua de Señas); sin embargo, las actuales políticas educativas promovidas por la Convención de los Derechos de las Personas con Discapacidad de las Naciones Unidas (2007), expresa en su articulado, políticas que aseguran al sordo su acceso a una educación bilingüe plena. Lo mismo que ha sido sustentado en múltiples investigaciones y estudios que demuestran que la lengua de señas tiene el valor lingüístico y expresivo a cualquier nivel de abstracción y que su aprendizaje temprano, favorece la comprensión y el desarrollo cognitivo. Además, existen estudios que han demostrado que el oralismo o la oralidad, como único medio para el aprendizaje del niño sordo, ha registrado resultados deficientes para el desarrollo pleno del sordo (Daniel, 2014).

Es aquí donde la sordedad toma relevancia para mi entendimiento porque en una sociedad donde se articula una educación bilingüe, se potencia la enseñanza de las lenguas (señas, escrita y oral), bien conjugadas entre sí. Pero es importante que desde el punto de vista 
cultural, todas las personas involucradas en la educación de sordos, permitan que la visión de "ser sordo" sean no solamente comprendida, sino también aceptada. Los sordos reclaman espacios para defender sus derechos y deberes: su protagonismo, su historia, su lengua y su identidad cultural. Permítase entonces a la persona "ser sordo o sorda". De manera que la cuasi utopía de los países latinoamericanos de una educación bilingüe que, dicho sea de paso, ha sido lograda en países avanzados, tales como Finlandia, Suecia e Inglaterra, entre otros; en donde, sordos profesionales imparten clases a sus pares y homólogos estudiantes sordos, se convierte en una realidad.

Para este logro existen una serie de situaciones que, a la luz de la teoría del iceberg, han sido consideradas como necesarias para el logro y evolución de una educación bilingüe, pero que quedan solapadas en el intento por construir este ideal. Tal es el caso de la formación superior de sordos, que tiene que constituirse en uno de los motores principales y articuladores de motivaciones para impulsar, en las sociedades modernas, la implementación de una educación bilingüe y de calidad en las cuales, los propios sordos se conviertan en docentes de sordos. De la misma manera que debe develarse el lado oculto de lo que la analogía del iceberg identifica como otras consideraciones del bilingüismo de los sordos (López, 2018).

Según lo aprendido en cultura de sordos, existen otras consideraciones que demuestran que la educación bilingüe de los sordos tiene mucho de la analogía de la teoría del Iceberg. Puesto que, lo que se observa en la actualidad, en los países en desarrollo, es sólo la punta del iceberg. O sea, una parte de la evolución de los componentes de una educación bilingüe. Todavía la parte sumergida del iceberg contiene elementos ocultos que son tan potentes que lastran la evolución de la educación, al ritmo deseado (García, 2015). Entre estas consideraciones del bilingüismo de los sordos, que son identificados en el iceberg, se encuentran: 1) el desarrollo de una suficiencia en ambas lenguas (profesores e intérpretes); 2) el desarrollo de una suficiencia en la Lengua, L1 en los Sordos (con adultos sordos); 3) el desarrollo de una suficiencia en la Lengua, L2 en los Sordos (con profesores oyentes señantes expertos o sordos adultos que manejen bien el español); y 4) el conocimiento y reconocimiento de la cultura sorda, su aprendizaje, aculturación o inculturación, que realice plenamente la inmersión de todos sus participantes (López, 2018).

Esto nos lleva al análisis del por qué existe un estancamiento en el desarrollo de una educación bilingüe en el sordo. Tal vez esta detención se deba a lo que sostiene García (2015, p. s/n) "como el cambio en lo estructural en la teoría del iceberg" quiere decir que las iniciativas para el cambio de un modelo clínico a uno social se están focalizando solo en algunos aspectos que, sin ser menores, no llegan a actuar sobre otros elementos del problema.

Pese que existe un gran interés por generar el cambio en la educación de sordos, esta no avanza al ritmo que la sociedad lo requiere y que los estudios avanzados de sordos lo indican, por los parámetros estructurales arcaicos que ejercen su influencia y lastre que ralentiza cualquier posibilidad de cambio. Siendo así, es pertinente esgrimir el desarrollo de políticas y planes que sean cónsono con la realidad contextualizada, lo que permite que, en el caso de los sordos, se logre una educación bilingüe, de calidad, pertinente y acorde a sus potenciales. Es observar y analizar el problema de la mala calidad de la educación de sordos en los países de la región, desde diversas perspectivas que conduzcan a soluciones puntuales, de carácter formativo y 
académico con innovación y desarrollo.

Significa, entonces, que deben establecerse, en las políticas educativas, el cómo, cuándo y qué debe resolverse para que los sordos puedan acceder a una educación bilingüe plena. Es considerar una serie de elementos que responden al mejoramiento del abordaje en la educación de sordo, elementos que ya han sido definidos por un sin número de investigaciones, entre las cuales puede mencionarse los estudios que demuestran que la lengua de señas tiene el valor lingüístico y expresivo a cualquier nivel de abstracción. Adicional a ello, el aprendizaje temprano de la lengua de señas favorece la comprensión y el desarrollo cognitivo. También, deben tenerse en cuenta otros estudios que indican que el oralismo, como único medio de aprendizaje, no ha dado los resultados esperados (Daniel, 2014). Lo que significa que una educación bilingüe tiene que potenciarse en la enseñanza de ambas lenguas de manera completa con todos los elementos que se conjugan entre sí y entre ellas, la lengua de señas y la oral nacional (escrita), la cual, en el caso de Panamá, sería el Castellano.

Aprovecho esta ocasión y este documento para responder a la pregunta de que si la educación de sordos en Latinoamérica ha tenido avances. Pues claro que sí ha habido avances y muy significativos, que no pueden negarse, pero estos adelantos aun siendo significativos, en cuanto a la participación de sordos en el contexto educativo y en la formación superior, todavía describen una situación de discriminación en algunos países de la región, que demuestra que tiene que mejorarse o cambiarse el planteamiento o modelos de atención de las personas sordas. Esta situación descrita obedece a que aún las personas sordas experimentan rechazos en el reconocimiento de su lengua de señas y que según Harlan Lane, en su obra "Cuando la mente escucha", nos relata que "los sordos aún cuando sean oralizados nunca dejan de utilizar ni cambian la lengua de señas por el habla" (Lane, 1984, p.67). Por otra parte, la dificultad que experimentan en el dominio de la lengua oral es, eminente, lo que puede ser solucionado si se implementa una propuesta de educación bilingüe.

Como lo indica Grosjean (1999), para que exista un programa de educación que se ajuste a los niveles de bilingüismo de los sordos, esta educación bilingüe necesita la integración de procesos sine qua non que permitirán y serán parte fundamental de su desarrollo (mente sana, entorno lingüístico y comunicación dialógica). Es evidentemente necesario que estos elementos permitan al "sordo la adquisición de las dos lenguas: la lengua de señas de la comunidad sorda (como primera lengua), y la lengua oral de la mayoría de los oyentes (segunda lengua)". "Para ello, el niño debe tener contacto con las dos comunidades lingüísticas y debe sentir la necesidad de aprender y utilizar ambas lenguas" (Grosjean, 1999, p.4).

Es interesante examinar el bilingüismo de los sordos a la luz de la analogía de la teoría del iceberg, porque al compararse lo que sucede en la educación bilingüe puede decirse que existen diversos elementos que conforman un programa bilingüe que, al no ser considerados, pueden ralentizar la evolución y dinámica del programa, porque lleva oculto situaciones que se encuentran inmersas en el tema y que a la consideración del bilingüismos lo que se observa muchas veces no expresa todo lo que concierne al tema. Tal es el ejemplo de la muy bien estudiada y discutida temática de la clase de cultura de sordos. Para los sordos, la cultura está establecida por valores, experiencias y otros elementos que son intrínsecos a la comunidad de sordos y que se encuentran ocultos, en el iceberg. Lo que, para muchos oyentes, en la superficie real del 
iceberg, que se sitúa en el contexto, los oyentes no le prestan el valor necesario a la cultura de sordos en su interactuación con las personas sordas $\mathrm{o}$, sencillamente, no conocen sobre cultura de sordos y eso dificulta muchas veces el trabajo (López, 2018). Esto es, que todos los profesionales que trabajen con y para las personas sordas deben aprender sobre cultura de sordos.

Finalmente, debo indicar que esta experiencia maravillosa denominada cultura de sordos ha enriquecido mi existencia y me ha permitido aportar significativamente un granito de arena en la formación de la traducción e interpretación en Lengua de Señas Panameñas, comentario que hago y no tiene nada que ver con este documento directamente, pero sí, con la participación de profesionales de intermediación lingüística, llamados traductores e intérpretes en Lengua de Señas, quienes forman parte del conjunto denominado comunidad señante, quienes son también participantes en la educación bilingüe de los sordos. Por ello, un programa de educación bilingüe es multifactorial y multidimensional, porque asegura que las personas sordas interactúen en la vida con y/o entre sordos $u$ oyentes utilizando, de manera automática, la lengua de señas, la lengua escrita y la lengua oral. Concluyo este documento puntualizando que, para esbozar un programa de educación bilingüe de sordos, se deben contemplar los siguientes elementos:

1. Programa de formación en Lengua de Señas desde el nivel maternal hasta el avanzado y experto, mediante la enseñanza de personas de la comunidad de sordos, sordos adultos que tengan claro la formación en lengua de señas.

2. Deben asistir a su programa de formación en Lengua Oral nacional, con profesores oyentes expertos en lengua de señas y en educación de sordos que enseñen a los sordos la lectura y escritura, lo mismo que los componentes lingüísticos, fonológicos, sintácticos, morfológicos, semánticos y pragmáticos de la lengua oral, su articulación - voz, y, por qué no, la lectura labio facial, así como también, la organización del lenguaje.

3. La enseñanza y comprensión lectora y escritora, tiene que permitir al sordo una competencia lingüística que asegure el entendimiento de una conciencia fonológica, lo mismo que el significado oculto de las palabras, la apropiación de conceptos para que los utilice en su discurso y en su nivel de registro de la lengua.

4. Las clases de lengua de señas ylengua oral no pueden estar integradas en un mismo salón bajo un mismo horario, no. Tienen que estar separadas y cada clase tiene su programación, objetivos competenciales y apuntan hacia el desarrollo de ambas lenguas, por separado. Como decía Graciela Alisedo, un mismo maestro no puede enseñarle al sordo lengua oral y lengua de señas, pues el sordo aprende ni chicha ni limonada, o sea, nada.

5. Para que una educación bilingüe obtenga buenos resultados tiene que realizarse con buenas prácticas. Esto significa que la familia tiene que participar en la educación bilingüe del sordo, y que la familia de los niños sordos tiene que aprender lengua de señas. Esto es un ideal y sería fantástico puesto que asegura la participación plena de los sordos. He conocido familias en las cuales todos aprendieron lengua de señas para que su miembro sordo pudiera sentirse integrado, aceptado y desarrollado, lo que efectivamente, contribuye al desarrollo cognitivo del sordo. 
6. La escuela en donde se desarrolle un programa de educación bilingüe tiene que contar con todos los recursos: equipos, materiales, mobiliarios, tecnología de punta y otros elementos o detalles adecuados a una educación bilingüe. Esto sugiere que el escenario educativo se transforme en un espacio vivificador, transformador y gestor del bilingüismo con todos sus integrantes: estudiantes sordos, oyentes, personal docente, técnico y administrativo, todos sin excepción tienen que ser bilingüe. Los centros de recursos para la formación bilingüe tienen en su haber elementos que contribuyen a generar competencia lingüística en las lenguas que conforman la propuesta: lengua de señas nacional, lengua escrita oral nacional, y otra lengua extranjera (por ejemplo, el inglés).

7. Por ello, los profesionales vinculados con la educación bilingüe de sordos, llámese maestros, traductores o intérpretes, entre otros, tienen que ser totalmente bilingües o poseer una suficiencia en ambas lenguas que permita que los sordos puedan desarrollarse, como se dice en buen panameño: "nadar como peces en el agua" en un ambiente cultural y lingüístico rico y lleno de experiencias maravillosas.

\section{REFERENCIAS}

Alba, A. Samaniego, I. (2018). Psicolingüística, Asignatura de la Licenciatura en Traducción e Interpretación en Lengua de Señas Panameñas. Panamá: UDELAS.

Alisedo, G. (1998). Comunicación y Desarrollo Lingüístico de las personas con discapacidad. Panamá: IPHE.

"British Broadcasting Corporation,
BBC"(2014). Cinco cosas que no sabe la sordera. Londres: Noticias el mundo "BBC Global News". Disponible en: http://www.bbc. com/mundo/noticias/2014/08/140808_salud_ cinco_cosas_que_no_sabe_sordera_Iv.

Cummings, J. (1983). Infancia y Aprendizaje: Interdependencia lingüística y desarrollo educativo de niños bilingües. Canadá: Universidad Alberta.

Daniel N, (2006, 2018). Formación de Conceptos en las Personas Sordas, Congreso Internacional de Educación Especial y Congresos Científico de la UDELAS. Panamá: UDELAS - IPHE.

Daniel N. Ramos, H. Barragán, E. Ferrer, E. y otros. (2014). La educación de Sordos en Panamá, Un abordaje para adecuar la oferta de los servicios en el marco de la Convención sobre los Derechos de las Personas con Discapacidad. Panamá: Instituto Panameño de Habilitación Especial, IPHE. Disponible en www.iphe.gob.pa/menu2/publicaci/sordo.pdf

García Pérez, J.B. (2015). Ellastre estructural en la educación y la analogía del iceberg. Universidad de Murcia: INED21. Disponible en: https://ined21.com/el-lastre-estructural-eneducacion-y-la-analogia-del-iceberg/

Federación Mundial de Sordos (2018). Our Work, 70 million deaf people. 300 sign languages. Unlimited potential. Disponible en: https://wfdeaf.org/our.work

Grosjean, F. (1999). ElDerecho del niño sordo a crecer bilingüe. Universidad de Neuchatel, Suiza. Barcelona: Difusord, Asociación de Difusión de la Comunidad Sorda.

Howard, N. (2017). Conferencia Internacionalización: Intérprete en el proceso de aculturación y enculturación - intérprete 
sordo. III Encuentro Latinoamericano de Traductores e Intérpretes y Guías Intérpretes para Sordociegos. WASLI: Panamá

Howard, N. Daniel, N. (2016). "Deafhood" (Sordismo): La Cultura de Sordos, Diversidad Global y Datos Recientes sobre Interpretación en Lengua de Señas. Panamá: UDELAS.

Lane, H. (1984). When the Mind hears, A history of the Deaf New York: Penguin Books.

López F. L.M. (2018). Cultura de Sordos: Creencias sobre personas sordas, Apuntes de la Asignatura de la Carrera de Licenciatura en Traducción e Interpretación en Lengua de Señas Panameñas, Panamá: UDELAS.

Matheus,J. (2018) Psicología Evolutiva. Asignatura de Traducción e Interpretación en Lengua de Señas Panameñas. Panamá: UDELAS. Documento Inédito policopiado

Psicolingüística CECAR (2018) Documento PDF - Psicolingüística, Dirección de Educación
Abierta y a Distancia, consultada el 11 de abril de 2018. Documento Inédito policopiado

Rodríguez F., A. García G., A y otros (2008). Deficiencia auditiva, Desarrollo psicoevolutivo y respuesta educativa. Madrid: EOS.

Sánchez, S. (2016) La Lingüística de las Lenguas de Señas como herramienta de reivindicación política: problemas ideológicos y epistemológicos. ReVEL, 14, 26. Disponible en www.revel.inf.br/esp

Stokoe, W. (1960). SignLanguageStructure (Estructura de la lengua de señas), Studies in LinguisticOccasionalPaper, Buffalo, NY: University of Buffalo.

Veinberg, S. (2002). La perspectiva socio antropológica de la sordera. Buenos Aires: Universidad de Buenos Aires. Disponible en: www.sancristobal.amgr.es/.../Veinberg perspectiva_socioantropologica_Sordera.pdf 